\title{
ON THE COMPUTATION OF FACTORIZATION INVARIANTS FOR AFFINE SEMIGROUPS
}

\author{
PEDRO GARCÍA-SÁNCHEZ, CHRISTOPHER O'NEILL, AND GAUTAM WEBB
}

\begin{abstract}
We present several new algorithms for computing factorization invariant values over affine semigroups. In particular, we give (i) the first known algorithm to compute the delta set of any affine semigroup, (ii) an improved method of computing the tame degree of an affine semigroup, and (iii) a dynamic algorithm to compute catenary degrees of affine semigroup elements. Our algorithms rely on theoretical results from combinatorial commutative algebra involving Gröbner bases, Hilbert bases, and other standard techniques. Implementation in the computer algebra system GAP is discussed.
\end{abstract}

\section{INTRODUCTION}

Let $\Gamma=\left\langle\alpha_{1}, \ldots, \alpha_{k}\right\rangle \subset \mathbb{N}^{d}$ be a finitely generated subsemigroup of $\mathbb{N}^{d}$, that is, an affine semigroup. We are interested in studying the factorization structure of elements $\gamma \in \Gamma$, that is, the distinct expressions of $\gamma$ as a sum of the generators $\alpha_{1}, \ldots, \alpha_{k}$. Most of the theory on nonunique factorization stems from the study of factorizations in integral domains; in this context, one can view factorizations of affine semigroup elements as factorizations of monomials in the corresponding monoid algebra $\mathbb{K}[\Gamma]$ for $\mathbb{K}$ a field. Often, factorization structure is examined using factorization invariants, which assign quantities to each element of $\Gamma$ (or to $\Gamma$ as a whole) that measure the failure of its factorizations to be unique [22].

Every affine semigroup $\Gamma$ is an FF-monoid, meaning that each element $\gamma \in \Gamma$ has only finitely many factorizations. As such, many factorization invariants can be computed explicitly using computer software [20], an attribute that has greatly aided their study [28]. On the other hand, the class of affine semigroups is sufficiently broad to exhibit varied factorization structure, and remains an interesting source of examples and an active area of study.

In this paper, we present new algorithms for computing several well-studied factorization invariants. After some preliminary definitions in Section 2, we present in Section 3 the first known algorithm for computing the delta set of an affine semigroup. Two distinct approaches are given (Algorithms 3.6 and 3.9), the latter of which introduces a novel connection between the delta set and Gröbner bases of homogeneous toric ideals (see Remark 3.12). Next, we give Algorithm 4.11 for dynamically computing catenary degrees of affine semigroup elements, in the style of several known algorithms for other factorization invariants [3]. We conclude with an improved algorithm for computing the tame degree of an affine semigroup in Section 5 .

Following the statement and proof of correctness of each algorithm, we discuss implementation in the GAP [17] package numericalsgps [14, including sample code and benchmark comparisons with existing algorithms. Whenever a Gröbner basis (Definition 2.9) or a Hilbert basis (Definition 2.3) must be computed, our implementations rely on trusted software packages like 4ti2 [1], Normaliz [5], and Singular [13].

Date: May 28, 2021. 
Notation. In what follows, let $\mathbb{N}=\{0,1,2, \ldots\}$ denote the set of non-negative integers. Fix a field $\mathbb{K}$, and let $\mathbb{K}\left[y_{1}, \ldots, y_{k}\right]$ denote the polynomial ring in commuting variables $y_{1}, \ldots, y_{k}$ with coefficients in $\mathbb{K}$. Lastly, for $z \in \mathbb{N}^{k}$ we use the shorthand $y^{z}=y_{1}^{z_{1}} \cdots y_{k}^{z_{k}} \in \mathbb{K}\left[y_{1}, \ldots, y_{k}\right]$.

\section{BACKGROUND}

Definition 2.1. A semigroup $\Gamma \subset \mathbb{N}^{d}$ is affine if it is finitely generated. If $\Gamma \subset \mathbb{N}$ and $\operatorname{gcd}(\Gamma)=1$, we say $\Gamma$ is a numerical semigroup.

Remark 2.2. Any affine semigroup $\Gamma$ is reduced, and thus has a unique generating set that is minimal with respect to containment. Throughout this paper, whenever we write $\Gamma=\left\langle\alpha_{1}, \ldots, \alpha_{k}\right\rangle$, we assume $\alpha_{1}, \ldots, \alpha_{k}$ are precisely the minimal generators of $\Gamma$.

Definition 2.3. Fix a matrix $A \in \mathbb{Z}^{k \times d}$. The set

$$
\Gamma=\left\{x \in \mathbb{N}^{d}: A x=0\right\}
$$

of nonnegative integer homogeneous solutions of $A$ forms an affine semigroup. The minimal generating set of $\Gamma$ (w.r.t. containment) is called the Hilbert basis of $A$, denoted $\mathcal{H}(\Gamma)$.

Definition 2.4. Fix an affine semigroup $\Gamma=\left\langle\alpha_{1}, \ldots, \alpha_{k}\right\rangle \subset \mathbb{N}^{d}$. The elements $\alpha_{1}, \ldots, \alpha_{k}$ comprising the unique minimal generating set of $\Gamma$ are called irreducible elements (or atoms). A factorization of $\gamma \in \Gamma$ is an expression

$$
\gamma=z_{1} \alpha_{1}+\cdots+z_{k} \alpha_{k}
$$

of $\gamma$ as a finite sum of atoms, which we denote by the $k$-tuple $z=\left(z_{1}, \ldots, z_{k}\right) \in \mathbb{N}^{k}$. Write $\mathrm{Z}_{\Gamma}(\gamma)$ for the set of factorizations of $\gamma \in \Gamma$, viewed as a subset of $\mathbb{N}^{k}$.

Since $\alpha_{1}, \ldots, \alpha_{k}$ generate $\Gamma$, the monoid homomorphism $\varphi_{\Gamma}: \mathbb{N}^{k} \rightarrow \Gamma$ given by

$$
\varphi_{\Gamma}\left(z_{1}, \ldots, z_{k}\right)=z_{1} \alpha_{1}+\cdots+z_{k} \alpha_{k}
$$

is surjective, and $\Gamma \cong \mathbb{N}^{k} / \operatorname{ker} \varphi_{\Gamma}$, where

$$
\operatorname{ker} \varphi_{\Gamma}=\left\{(z, w) \in \mathbb{N}^{k} \times \mathbb{N}^{k}: \varphi_{\Gamma}(z)=\varphi_{\Gamma}(w)\right\}
$$

denotes the kernel of $\varphi_{\Gamma}$. Notice that ker $\varphi_{\Gamma}$ is a congruence on $\mathbb{N}^{k}$ (i.e. an equivalence relation that is closed under translation), and thus it is finitely generated (see 229]). In particular, there exists a finite set $\rho \subseteq \operatorname{ker} \varphi_{\Gamma}$ such that the smallest congruence containing $\rho$ is $\operatorname{ker} \varphi_{\Gamma}$ (equivalently, $\operatorname{ker} \varphi_{\Gamma}$ equals the intersection of all congruences containing $\rho$ ).

Definition 2.5. A minimal presentation of $\Gamma$ is a generating set $\rho$ of the congruence $\operatorname{ker} \varphi_{\Gamma}$ that is minimal with respect to containment. The Betti elements of $\Gamma$ are elements of the form $\varphi_{\Gamma}(z)$ for $(z, w) \in \rho$ (this is independent of the minimal presentation chosen).

Definition 2.6. Fix $\Gamma=\left\langle\alpha_{1}, \ldots, \alpha_{k}\right\rangle \subset \mathbb{N}^{d}$, and let $A=\left(\alpha_{1}|\cdots| \alpha_{k}\right)$ denote the matrix with columns $\alpha_{1}, \ldots, \alpha_{k}$. The Graver basis of $A$ (or the Graver basis of $\Gamma$ ) is the Hilbert basis of the matrix $(A \mid-A)$. 
Remark 2.7. The Graver basis of a semigroup $\Gamma$ contains every minimal presentation of $\Gamma$, but is typically orders of magnitude larger. Even for numerical semigroups with 3 minimal generators, whose minimal presentations have at most 3 relations, the Graver basis can be arbitrarily large. As such, algorithms relying on a proper subset of the Graver basis (such as a minimal presentation or a Gröbner basis) are preferred whenever possible.

Example 2.8. Let $\Gamma=\langle 3,4,5\rangle \subseteq \mathbb{N}$. The minimal presentation of $\Gamma$ is

$$
\rho=\{((1,0,1),(0,2,0)),((2,1,0),(0,0,2)),((3,0,0),(0,1,1))\},
$$

as computed in [31, Example 8.23]. In this case, $\rho$ is the only minimal presentation of $\Gamma$, though minimal presentations need not be unique in general. For comparison, the Graver basis of $\Gamma$ consists of (upon omitting symmetry) the pairs

$$
\begin{aligned}
& ((1,0,1),(0,2,0)),((1,3,0),(0,0,3)),((2,1,0),(0,0,2)), \\
& ((3,0,0),(0,1,1)),((4,0,0),(0,3,0)),((5,0,0),(0,0,3)) .
\end{aligned}
$$

We associate to $\Gamma$ an ideal $I_{\Gamma} \subset \mathbb{K}\left[y_{1}, \ldots, y_{k}\right]$ defined as follows. Consider the polynomial $\operatorname{ring} \mathbb{K}\left[t_{1}, \ldots, t_{d}\right]$, and more specifically the subring $\mathbb{K}[\Gamma]=\bigoplus_{\gamma \in \Gamma} \mathbb{K} t^{\gamma}$. In this setting, $\varphi_{\Gamma}$ has a polynomial ring counterpart, namely the ring homomorphism

$$
\psi_{\Gamma}: \mathbb{K}\left[y_{1}, \ldots, y_{k}\right] \rightarrow \mathbb{K}[\Gamma]
$$

determined by $\psi_{\Gamma}\left(y_{i}\right)=t^{\alpha_{i}}$. The defining ideal of $\Gamma$ is the kernel

$$
I_{\Gamma}=\operatorname{ker} \psi_{\Gamma} \subset \mathbb{K}\left[y_{1}, \ldots, y_{k}\right] .
$$

It is well known that a set $\rho \subset \operatorname{ker} \varphi_{\Gamma}$ generates $\operatorname{ker} \varphi_{\Gamma}$ as a congruence if and only if

$$
\left\{y^{z}-y^{w}:(z, w) \in \rho\right\} \subset I_{\Gamma}
$$

generates $I_{\Gamma}$, and that $\rho$ is a minimal presentation for $\Gamma$ precisely when the above set irredundantly generates $I_{\Gamma}$ (see [24]).

We conclude this section with a brief discussion of Gröbner bases, which are standard in computational commutative algebra; the unfamiliar reader is encouraged to consult [12] for a more thorough overview.

Definition 2.9. Fix a monomial order $\preceq$ on $\mathbb{K}\left[y_{1}, \ldots, y_{k}\right]$ (that is, a total ordering of the monomials containing the usual partial ordering by divisibility). Given $f \in \mathbb{K}\left[y_{1}, \ldots, y_{k}\right]$, denote by $\operatorname{In}_{\preceq}(f)$ the initial term of $f$ with respect to $\preceq$. A Gröbner basis of an ideal $I \subset \mathbb{K}\left[y_{1}, \ldots, y_{k}\right]$ with respect to $\preceq$ is a set $G=\left\{g_{1}, \ldots, g_{r}\right\}$ generating $I$ such that $\left\langle\operatorname{In}_{\preceq}\left(g_{1}\right), \ldots, \operatorname{In}_{\preceq}\left(g_{r}\right)\right\rangle=\left\langle\left\{\operatorname{In}_{\preceq}(f): f \in I\right\}\right\rangle$.

Remark 2.10. Minimal presentations can be computed using Gröbner bases. Set

$$
J=\left\langle y_{1}-t^{\alpha_{1}}, \ldots, y_{k}-t^{\alpha_{k}}\right\rangle \subseteq \mathbb{K}\left[y_{1}, \ldots, y_{k}, t_{1}, \ldots, t_{d}\right],
$$

and observe that $I_{\Gamma}=J \cap \mathbb{K}\left[y_{1}, \ldots, y_{k}\right]$. To compute a generating set for $I_{\Gamma}$, one can compute a Gröbner basis $G$ for $J$ with respect to any monomial ordering $\preceq$ satisfying $y_{i} \preceq t_{j}$ for all possible $i$ and $j$, and then calculate $G \cap \mathbb{K}\left[y_{1}, \ldots, y_{k}\right]$. 


\section{The DELTA SET}

In this section, we introduce Algorithms 3.6 and 3.9, the first known algorithms for computing the delta set of any affine semigroup, as well as proofs of correctness (Theorems 3.8 and 3.10, respectively). Both algorithms have Theorem 3.5 at their core, but each uses a different method for computing the necessary ideal generating sets.

Definition 3.1. Fix an affine semigroup $\Gamma=\left\langle\alpha_{1}, \ldots, \alpha_{k}\right\rangle \subset \mathbb{N}^{d}$ and $\gamma \in \Gamma$. Given $z \in Z_{\Gamma}(\gamma)$, the length of $z$ is the number $|z|=z_{1}+\cdots+z_{k}$ of irreducibles in $z$. The length set of $\gamma$ is

$$
\mathrm{L}_{\Gamma}(\gamma)=\left\{z_{1}+z_{2}+\cdots+z_{k}: z \in \mathrm{Z}_{\Gamma}(\gamma)\right\},
$$

the set of factorization lengths. Writing $L_{\Gamma}(\gamma)=\left\{\ell_{1}<\cdots<\ell_{m}\right\}$, the delta set of $\gamma$ is the set

$$
\Delta(\gamma)=\left\{\ell_{i+1}-\ell_{i}: 1 \leq i<m\right\}
$$

of successive differences of factorization lengths. The delta set of $\Gamma$ is $\Delta(\Gamma)=\bigcup_{\gamma \in \Gamma} \Delta(\gamma)$.

Theorem 3.2 follows from [8, Corollary 2.4, Thoerem 2.5], and describes which delta set elements can be easily recovered from a minimal presentation.

Theorem 3.2. If $\Gamma=\left\langle\alpha_{1}, \ldots, \alpha_{k}\right\rangle \subset \mathbb{N}^{d}$ and $\rho \subset\left(\mathbb{N}^{k}\right)^{2}$ is a minimal presentation of $\Gamma$, then

$$
\min \Delta(\Gamma)=\operatorname{gcd}(\{|z|-|w|:(z, w) \in \rho\}),
$$

$$
\max \Delta(\Gamma)=\max \left\{\max \Delta\left(z_{1} \alpha_{1}+\cdots+z_{k} \alpha_{k}\right) \mid(z, w) \in \rho\right\}
$$

and

$$
\Delta(\Gamma) \subseteq[\min \Delta(\Gamma), \max \Delta(\Gamma)] \cap \min \Delta(\Gamma) \mathbb{Z} .
$$

In particular, $\min \Delta(\Gamma)=\operatorname{gcd} \Delta(\Gamma)$, and both $\min \Delta(\Gamma)$ and $\max \Delta(\Gamma)$ can be quickly recovered from $\rho$.

Example 3.3. Let us return to $\Gamma=\langle 3,4,5\rangle$ from Example 2.8. According to Theorem 3.2 , the minimum and maximum of $\Delta(\Gamma)$ are determined by $\Delta(8), \Delta(9)$ and $\Delta(10)$. The only factorizations of 8 are $(0,2,0)$ and $(1,0,1)$, so $\Delta(8)=\emptyset$. We can obtain $\Delta(9)=\Delta(10)=\{1\}$ similarly. As such, $\Delta(\Gamma)=\{1\}$, since in this case $\min \Delta(\Gamma)=\max \Delta(\Gamma)$. In general, however, Theorem 3.2 only yields an interval containing $\Delta(\Gamma)$.

Remark 3.4. The primary difficulty in computing $\Delta(\Gamma)$ for a general affine semigroup $\Gamma$ is ensuring that a given value does not occur in $\Delta(\Gamma)$. Indeed, some elements of $\Delta(\Gamma)$ may only occur in the delta sets of a small (finite) number of semigroup elements. For example, if $\Gamma=\langle 17,33,53,71\rangle \subset \mathbb{N}$, then $6 \in \Delta(\Gamma)$ only occurs in $\Delta(266), \Delta(283)$, and $\Delta(300)$.

As such, although it is computationally feasible to compute the delta set of any single element of $\Gamma$ (since each has only finitely many factorizations), this cannot be accomplished for all of the (infinitely many) elements of $\Gamma$. To date, all existing delta set algorithms are limited to numerical semigroups, and act by bounding the semigroup elements for which "new" elements of $\Delta(\Gamma)$ can occur, thus restricting computation to a finite list of semigroup elements; see [3] for more detail.

One of the primary benefits of Algorithm 3.6 is that it does not rely on computing delta sets of individual semigroup elements. As such, both of our algorithms are generally much faster than existing algorithms for numerical semigroups; see Examples 3.13 and 3.14 . 
We now state Theorem 3.5, the main theoretical result used in Algorithm 3.6.

Theorem 3.5 ([27, Theorem 4.8]). Suppose $\Gamma=\left\langle\alpha_{1}, \ldots, \alpha_{k}\right\rangle \subset \mathbb{N}^{d}$. The ideals

$$
I_{j}=\left\langle y^{z}-y^{w}: z, w \in \mathbf{Z}_{\Gamma}(\gamma), \gamma \in \Gamma, \text { and }|| w|-| z|| \leq j\right\rangle \subset \mathbb{K}\left[y_{1}, \ldots, y_{k}\right]
$$

for $j \geq 0$ form an ascending chain

$$
I_{0} \subset I_{1} \subset I_{2} \subset \cdots
$$

in which $I_{j-1} \subsetneq I_{j}$ if and only if $j \in \Delta(\Gamma)$.

Algorithm 3.6 obtains the delta set of a given affine semigroup $\Gamma=\left\langle\alpha_{1}, \ldots, \alpha_{k}\right\rangle \subset \mathbb{N}^{d}$ by computing each ideal defined in Theorem 3.5. The generating set of $I_{j}$ appearing in Theorem 3.5 is determined by (integer solutions to) a system of linear inequalities, and thus can be computed using a Hilbert basis. For example, by inserting a slack variable to transform the single inequality to an equality, it suffices to compute a Hilbert basis for the matrix $A$ below. Each element $x=(z, w, i) \in \mathbb{N}^{k} \times \mathbb{N}^{k} \times \mathbb{N}$ of the Hilbert basis with $i \leq j$ yields a binomial generator $y^{z}-y^{w}$ for $I_{j}$ by Lemma 3.7 .

$$
A=\left(\begin{array}{ccccccc}
\alpha_{11} & \cdots & \alpha_{1 k} & -\alpha_{11} & \cdots & -\alpha_{1 k} & 0 \\
\vdots & \ddots & \vdots & \vdots & \ddots & \vdots & \vdots \\
\alpha_{d 1} & \cdots & \alpha_{d k} & -\alpha_{d 1} & \cdots & -\alpha_{d k} & 0 \\
1 & \cdots & 1 & -1 & \cdots & -1 & -1
\end{array}\right)
$$

Once each generating set has been computed, successive ideals $I_{j-1} \subset I_{j}$ can be checked for strict containment by computing a reduced Gröbner basis for each ideal (with respect to the same term order) and then comparing. The final step in the algorithm is locating the maximal value of $j$, which by Theorem 3.2 can be obtained by computing a minimal presentation for $\Gamma$. Note that each element of $\Delta(\Gamma)$ is a multiple of $\min \Delta(\Gamma)$, which by Theorem 3.2 can also be obtained from a minimal presentation for $\Gamma$.

We are now ready to state Algorithm 3.6.

Algorithm 3.6. Computes the delta set of an affine semigroup $\Gamma=\left\langle\alpha_{1}, \ldots, \alpha_{k}\right\rangle \subset \mathbb{N}^{d}$.

function DeltaSetOfAffineSemigroup $(\Gamma)$

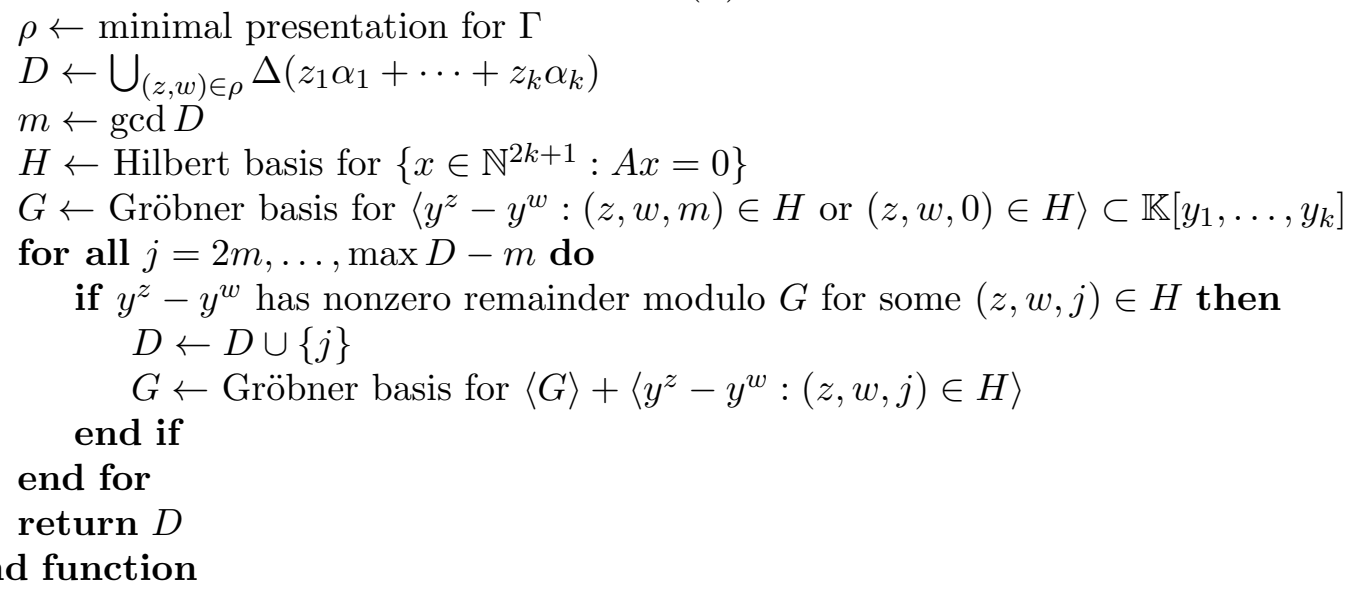

We now give a proof that Algorithm 3.6 gives the correct output. 
Lemma 3.7. Resume notation from Theorem 3.5 and Algorithm 3.6. The ideal

$$
J_{j}=\left\langle y^{z}-y^{w}:(z, w, i) \in H, i \leq j\right\rangle \subset \mathbb{K}\left[y_{1}, \ldots, y_{k}\right]
$$

computed in Algorithm 3.6 coincides with the ideal $I_{j}$ from Theorem 3.5 for all $j \geq 0$.

Proof. For each $i \leq j$ and $(z, w, i) \in H$, it is clear that $y^{z}-y^{w} \in I_{j}$, so $J_{j} \subset I_{j}$. Conversely, if $(z, w, i),\left(z^{\prime}, w^{\prime}, i^{\prime}\right) \in\langle H\rangle$ with $y^{z}-y^{w}, y^{z^{\prime}}-y^{w^{\prime}} \in I_{j}$, then

$$
y^{z^{\prime}}\left(y^{z}-y^{w}\right)+y^{w}\left(y^{z^{\prime}}-y^{w^{\prime}}\right)=y^{z+z^{\prime}}-y^{w+w^{\prime}} \in I_{j},
$$

which is the binomial corresponding to $(z, w, i)+\left(z^{\prime}, w^{\prime}, i^{\prime}\right) \in\langle H\rangle \subset \mathbb{N}^{2 k+1}$. This means the generating set for $I_{j}$ given in Theorem 3.5 can be restricted to the binomials corresponding to elements of the Hilbert basis $H$, so $I_{j} \subset J_{j}$ as well.

Theorem 3.8. For any affine semigroup $\Gamma \subset \mathbb{N}^{d}$, Algorithm 3.6 returns $\Delta(\Gamma)$.

Proof. Resume notation from Theorem 3.5 and Algorithm 3.6. Theorem 3.2 implies that $m=\min \Delta(\Gamma)$, and that only multiples of $m$ can appear in $\Delta(\Gamma)$. Lemma 3.7 ensures each loop iteration computes the ideal $I_{j}$ for some multiple $j$ of $m$, and appends $j$ to $D$ if $I_{j-1} \subsetneq I_{j}$. Applying Theorem 3.5 completes the proof.

Algorithm 3.9 uses an alternative approach to compute generators for the ideals $I_{j}$ in Theorem 3.5. Given $\alpha \in \mathbb{N}^{d}$, denote by $(1, \alpha)$ the element in $\mathbb{N}^{d+1}$ whose first coordinate is 1 and whose remaining coordinates are those in $\alpha$. For $\Gamma=\left\langle\alpha_{1}, \ldots, \alpha_{k}\right\rangle \subset \mathbb{N}^{d}$, set

$$
\Gamma_{H}=\left\langle(1,0),\left(1, \alpha_{1}\right), \ldots,\left(1, \alpha_{k}\right)\right\rangle \subset \mathbb{N}^{d+1} .
$$

There is a tight relation between factorizations in $\Gamma_{H}$ and those in $\Gamma$ [21]. Observe that

$$
\mathrm{I}_{\Gamma_{H}}=\left\langle t^{|w|-|z|} y^{z}-y^{w}: z, w \in \mathrm{Z}(\gamma), \gamma \in \Gamma,|z| \leq|w|\right\rangle \subset \mathbb{K}\left[t, y_{1}, \ldots, y_{k}\right] .
$$

Algorithm 3.9 exploits this idea to compute the generators $I_{j}$ in Theorem 3.5. As there is no need to compute a Graver basis (see Remark 2.7), Algorithm 3.9 is better equipped than Algorithm 3.6 to handle input with large generators (see Example 3.13 .

Algorithm 3.9. Computes the delta set of an affine semigroup $\Gamma=\left\langle\alpha_{1}, \ldots, \alpha_{k}\right\rangle \subset \mathbb{N}^{d}$.

function DeltaSetOfAffineSemigroup $(\Gamma)$

$\rho \leftarrow$ minimal presentation for $\Gamma_{H}=\left\langle(1,0),\left(1, \alpha_{1}\right), \ldots,\left(1, \alpha_{k}\right)\right\rangle$

$G \leftarrow$ reduced lex Gröbner basis for $\left\langle t^{i} y^{z}-t^{j} y^{w}:((i, z),(j, w)) \in \rho\right\rangle \subset \mathbb{K}\left[t, y_{1}, \ldots, y_{k}\right]$

return $\left\{j: t^{j} y^{z}-y^{w} \in G\right\}$

\section{end function}

Theorem 3.10. Fix $\Gamma=\left\langle\alpha_{1}, \ldots, \alpha_{k}\right\rangle \subset \mathbb{N}^{d}$ an affine semigroup. Let $G$ denote a reduced Gröbner basis for $\mathrm{I}_{\Gamma_{H}}$ with respect to any lexicographic term order satisfying $t>y_{i}$ for each $i \leq k$, and for $j \geq 0$ let

$$
J_{j}=\left\langle y^{z}-y^{w}: t^{i} y^{z}-y^{w} \in G, i \leq j\right\rangle .
$$

We have $I_{j}=J_{j}$ for each $j \geq 0$. 
Proof. Each generator of $J_{j}$ certainly lies in $I_{j}$, so $J_{j} \subset I_{j}$. Conversely, fix $y^{z}-y^{w} \in I_{j}$ with $|z| \leq|w|$, and let $i=|w|-|z| \leq j$. By definition, $t^{i} y^{z}-y^{w} \in \mathrm{I}_{\Gamma_{H}}$, so performing polynomial long division by $G$ yields a remainder of 0 . Since $G$ was computed using a lex term order, the only elements of $G$ used in the division algorithm have leading term dividing $t^{i} y^{z}$, and thus correspond to generators of $J_{j}$. As such, $I_{j} \subset J_{j}$. The final claim follows from $G$ being reduced, since the initial term $t^{j} y^{z}$ of each $t^{j} y^{z}-y^{w} \in G$ cannot be divisible by the leading term of any other elements of $G$, including those corresponding to generators of $J_{j-1}$.

Corollary 3.11. Fix $\Gamma=\left\langle\alpha_{1}, \ldots, \alpha_{k}\right\rangle \subset \mathbb{N}^{d}$ an affine semigroup. If $G$ is a reduced Gröbner basis for $\mathrm{I}_{\Gamma_{H}}$ with respect to any lexicographic term order satisfying $t>y_{i}$ for $i \leq k$, then

$$
\Delta(\Gamma)=\left\{j: t^{j} y^{z}-y^{w} \in G\right\} .
$$

Remark 3.12. In addition to ensuring the correctness of Algorithm 3.9, Corollary 3.11 is also interesting from a theoretical perspective, as it expresses the delta set in terms of Gröbner bases of homogeneous toric ideals. The authors are optimistic that this novel connection will spur interest in the delta set from members of the computational algebra community.

3.1. Implementation and benchmarks. We implemented both algorithms above in GAP to compare their performance to existing methods. In our implementation of Algorithm 3.6. we used Normaliz [5] through NormalizInterface [23] for the calculation of the Hilbert basis $H$, and Singular [13] via the GAP package singular [11] for the computation of successive Gröbner bases. Although we did some experiments with 4ti2 [1] via [2 for the calculation of $H$, the resulting implementation was slower than with normaliz; additionally, one cannot use 4ti2 for Gröbner basis computations here since the ideals are not toric.

Example 3.13. For numerical semigroups, the algorithm used in the numericalsgps [14] package is the one presented in [3], which was the fastest known prior to those given above. Though it only works with numerical semigroups, we can still compare how it behaves against Algorithm 3.6. A log-time algorithm has also been given for numerical semigroups with 3 minimal generators [18, but the techniques used therein do not easily generalize.

In examples with "small" generators, Algorithm 3.6 outperforms DeltaSetOfNumericalSemigroup (the current implementation in numericalsgps). For instance, the calculation of $\Delta(\langle 101,123,147,199\rangle)$ takes $550 \mathrm{~ms}$ with the current implementation, compared to $50 \mathrm{~ms}$ with Algorithm 3.6. Additionally, the current implementation takes $415263 \mathrm{~ms}$ to compute $\Delta(\langle 101,123,147,199,210\rangle)$, while Algorithm 3.6 accomplishes this task in under 4 seconds.

For semigroups with large generators, the situation can differ. Choosing, for instance, $\Gamma=\langle 1001,1211,1421,1631,2841\rangle$, DeltaSet0fNumericalSemigroup computes $\Delta(\Gamma)$ in less than two minutes, whereas the processes running Normaliz completed Algorithm 3.6 in around three minutes, even when run with SCIP [15] (4ti2 was killed after half an hour).

One of the advantages of Algorithm 3.9 is that one can use either Singular (eliminate()) or 4ti2 (groebner()) to compute a Gröbner basis for $\mathrm{I}_{\Gamma_{H}}$, with comparable performance.

Example 3.14. All delta sets appearing in Example 3.13 were computed with Algorithm 3.9 in less than $20 \mathrm{~ms}$. Even for significantly larger examples such as

$$
\begin{gathered}
\Gamma=\langle 550,1060,1600,1781,4126,4139,4407,5167,6073,6079,6169,7097,7602,8782,8872\rangle, \\
\Delta(\Gamma)=\{1,2,3,4,5,6,7,8,9,10,11,12,13,14,15,16,17,19\} \text { computes in less than a minute. }
\end{gathered}
$$




\section{The CATENARY DEGREE: A DYNAMIC ALGORITHM}

The catenary degree is a factorization invariant that measures how spread out the factorizations of an element are. Recent results prove the catenary degree is eventually periodic for numerical semigroups [10, and a natural generalization is given for affine semigroups [27, but neither result gives a concrete bound for the start of periodic behavior (in contrast to similar results for several other invariants; see [28, Remark 5.11]). In this section, we give Algorithm 4.11 for computing the catenary degree of affine semigroup elements using dynamic programming, answering [28, Problem 5.12]. The primary motivation for such an algorithm is to aid the investigation of concrete bounds for the aforementioned eventual behavior results, and our approach mirrors several existing algorithms for other factorization invariants [3].

Definition 4.1. Fix $\Gamma=\left\langle\alpha_{1}, \ldots, \alpha_{k}\right\rangle \subset \mathbb{N}^{d}$ and $\gamma \in \Gamma$. The greatest common divisor of $z, w \in \mathbf{Z}(\gamma)$ is given by

$$
\operatorname{gcd}(z, w)=\left(\min \left(z_{1}, w_{1}\right), \ldots, \min \left(z_{k}, w_{k}\right)\right) \in \mathbb{N}^{k},
$$

and the distance between $z$ and $w$ (or the weight of $(z, w)$ ) is defined as

$$
\mathrm{d}(z, w)=\max (|z-\operatorname{gcd}(z, w)|,|w-\operatorname{gcd}(z, w)|) .
$$

Given $z, w \in \mathbf{Z}(\gamma)$ and $N \geq 0$, an $N$-chain from $z$ to $w$ is a sequence $z_{0}, \ldots, z_{r} \in \mathbf{Z}(\gamma)$ of factorizations of $\gamma$ such that (i) $z_{0}=z$, (ii) $z_{r}=w$, and (iii) $\mathrm{d}\left(z_{i-1}, z_{i}\right) \leq N$ for all $i \leq r$. The weight of $z_{0}, \ldots, z_{r}$ is the smallest $N$ such that $z_{0}, \ldots, z_{r}$ is an $N$-chain (or, equivalently, the largest distance between successive factorizations). The catenary degree of $\gamma$, denoted c $(\gamma)$, is the smallest $N$ such that there exists an $N$-chain between any two factorizations of $\gamma$.

Example 4.2. Consider the numerical semigroup $\Gamma=\langle 11,36,39\rangle$. The left-hand picture in Figure 1 depicts Z(450) and all pairwise distances. Any two factorizations of 450 are connected by a 16 -chain; one such chain between $z=(6,2,8)$ and $w=(24,3,2)$ is depicted with bold red edges. Every 16-chain between $z$ and $w$ contains the bottom edge, so $c(450)=16$.

The catenary degree can also be computed by examining edges with weight at most 16 , as depicted in the right-hand picture of Figure 1. Removing edges labeled 8 and 12 yields a (minimal total weight) spanning tree, at which point Lemma 4.5 implies $\mathrm{c}(450)=16$.

The graph theoretic description of the catenary degree evident in Example 4.2 is one of the core structures used in Algorithm 4.11. We now make this definition explicit.

Definition 4.3. Given $\gamma \in \Gamma$, the catenary graph of $\gamma$ is the complete graph $K_{\gamma}$ with $V\left(K_{\gamma}\right)=\mathrm{Z}(\gamma)$ whose edges are labeled by the distance function $\mathrm{d}(-,-)$.

To compute the catenary degree of an element $\gamma \in \Gamma$, one typically first computes $\mathbf{Z}(\gamma)$, and compiles a list of edges in $K_{\gamma}$ sorted by distance. From this list of edges, a minimal connected graph is constructed one edge at a time, starting with the lowest weight (one can use Kruskal's Algorithm [26] for this). The final edge weight equals the catenary degree. One of the primary bottlenecks in this procedure is listing and sorting the edges of $K_{\gamma}$, since for large $\gamma$ most edges have weight higher than the catenary degree and thus are never used.

The main goal of Algorithm 4.11 is to reduce the number of edges by remembering which edges were redundant for smaller elements. This is achieved by storing, for each $\gamma \in \Gamma$, a 

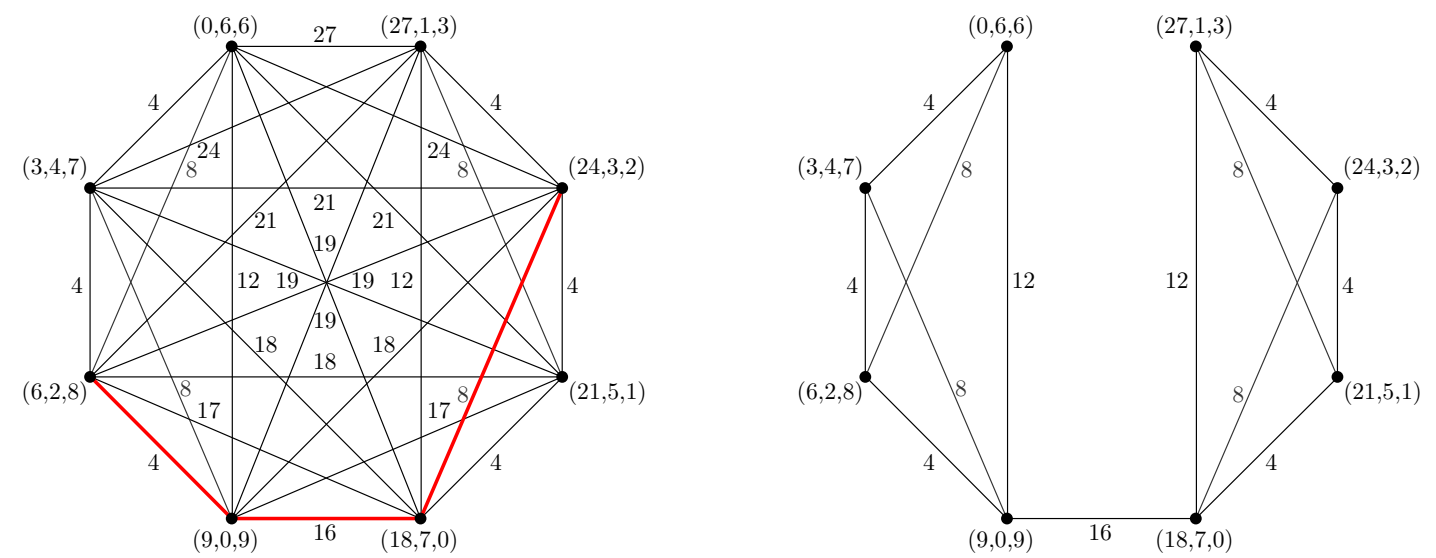

Figure 1. The catenary graph $K_{450}$ in $\Gamma=\langle 11,36,39\rangle$ from Example 4.2 . Both graphics were created using the computer algebra system SAGE 33 .

spanning tree of the graph $K_{\gamma}$ with minimum weight (Definition 4.4), from which the catenary degree can be easily recovered (Lemma 4.5). Indeed, the number of edges of $K_{\gamma}$ is quadratic in $\left|V\left(K_{\gamma}\right)\right|$, whereas the number of edges of a spanning tree is linear in $\left|V\left(K_{\gamma}\right)\right|$.

Definition 4.4. Let $G$ be an undirected graph with integer edge labels. We say a chain $q=v_{0}, v_{1}, \ldots, v_{r} \in V(G)$ has minimum weight if $q$ has minimum weight among all chains in $G$ connecting $v_{0}$ and $v_{r}$. A spanning tree $T$ of $G$ has minimum weight if the unique path between any two vertices $v_{0}$ and $v_{r}$ in $T$ is a minimum weight path in $G$.

Lemma 4.5. If $T$ is a minimum weight spanning tree of the catenary graph of $\gamma \in \Gamma$, then $\mathrm{c}(\gamma)$ equals the highest edge weight in $T$.

Proof. Immediate upon unraveling definitions.

The term "minimum weight spanning tree" usually refers to a spanning tree that minimizes edge weight sums. Proposition 4.6 verifies that our definition is equivalent to the usual one.

Proposition 4.6. Fix a graph $G$, a spanning tree $T \subset G$ with $E(T)=\left\{e_{1}, \ldots, e_{r}\right\}$, and a weight function $w$ on the edges of $G$. If $w\left(e_{1}\right)+\cdots+w\left(e_{r}\right)$ is minimal among all spanning trees of $G$, then $T$ is a minimum weight spanning tree of $G$.

Proof. Let $p$ denote a minimum weight chain $v_{0}, v_{1}, \ldots, v_{r} \in V(G)$, and let $q$ denote the unique path in $T$ from $v_{0}$ to $v_{r}$. For $i \leq r$, let $q_{i}$ denote the unique path in $T$ connecting $v_{i-1}$ and $v_{i}$. Concatenating the chains $q_{1}, q_{2}, \ldots, q_{r}$ produces a chain $q^{\prime}$ in $T$ connecting $v_{0}$ and $v_{r}$. Since $q$ is the unique path in $T$ connecting $v_{0}$ and $v_{r}$, every edge in $q$ must also be in $q^{\prime}$. In particular, the edge $e$ of highest weight in $q$ must lie in one of the chains $q_{m}$. As such, if $w(p)<w(q)$, then $w\left(v_{m-1}, v_{m}\right) \leq w(p)<w(q)=w(e)$, so deleting $e$ from $T$ and adding $\left(v_{m-1}, v_{m}\right)$ produces a spanning tree of lesser weight. This completes the proof.

Now that we have identified minimum weight spanning trees as sufficient for computing the catenary degree, it remains to see how a minimum weight spanning tree for $K_{\gamma}$ can be constructed from minimum weight spanning trees of its divisors. We accomplish this task 
using cover morphisms (Definition 4.7), which played a crucial role in the dynamic algorithms presented in [3]. The key observation is that cover morphisms also preserve edge weights when applied to adjacent factorizations in $K_{\gamma-\alpha_{i}}$ (Remark 4.8).

Definition 4.7. A cover morphism is a map $\psi_{i}: K_{\gamma-\alpha_{i}} \rightarrow K_{\gamma}$ that acts on the vertex set $V\left(K_{\gamma-\alpha_{i}}\right)=\mathrm{Z}\left(\gamma-\alpha_{i}\right)$ by incrementing the $i$-th coordinate of each factorization.

Remark 4.8. When applied to a pair $(z, w)$ of factorizations in $K_{\gamma-\alpha_{i}}$, the cover morphism $\psi_{i}$ applies the same translation to both $z$ and $w$, so the resulting factorizations $\left(z^{\prime}, w^{\prime}\right)$ satisfy

$$
z^{\prime}-\operatorname{gcd}\left(z^{\prime}, w^{\prime}\right)=z-\operatorname{gcd}(z, w) \quad \text { and } \quad w^{\prime}-\operatorname{gcd}\left(z^{\prime}, w^{\prime}\right)=w-\operatorname{gcd}(z, w) .
$$

In particular, $\mathrm{d}\left(z^{\prime}, w^{\prime}\right)=\mathrm{d}(z, w)$, so $\psi_{i}$ preserves edge weights (stated another way, $\psi_{i}$ defines an isometric embedding of $K_{\gamma-\alpha_{i}}$ into $\left.K_{\gamma}\right)$. Additionally, an edge $\left(z^{\prime}, w^{\prime}\right) \in E\left(K_{\gamma}\right)$ lies in the image of $\psi_{i}$ precisely when $i \in \operatorname{supp}\left(z^{\prime}\right) \cap \operatorname{supp}\left(w^{\prime}\right)$. Here, $\operatorname{supp}(z)$ denotes the support of $z$, that is, the set of indices of the nonzero coordinates of $z$.

As a final step, Theorem 4.10 implies that in addition to edges arising from the minimum weight spanning trees of $K_{\gamma-\alpha_{1}}, \ldots, K_{\gamma-\alpha_{k}}$, some supplementary edges from the Graver basis of $\Gamma$ are needed to obtain a minimum weight spanning tree for $K_{\gamma}$. The edges from the Graver basis of $\Gamma$ can be thought of as a base case for the dynamic process, in the sense that every edge used in a minimum weight spanning tree is a translation of a Graver basis element. Although the Graver basis of $\Gamma$ is computationally expensive (albeit necessary; see Example 4.9), it only needs to be calculated once for the monoid.

Example 4.9. Although a minimal presentation for $\Gamma$ produces enough edges via translation to connect $K_{\gamma}$ for every $\gamma \in \Gamma$, these edges are not sufficient for computing catenary degrees. For example, in the numerical semigroup $\Gamma=\langle 11,36,39\rangle$ from Example 4.2 , the only edge $e=((9,7,0),(0,0,9))$ in $K_{351}$ with weight $\mathrm{c}(351)=16$ does not appear in any minimal presentation. In fact, the weight 16 edge in Figure 1 is a translation of $e$. It is this phenomenon that forces Algorithm 4.11 to rely on a Graver basis for $\Gamma$.

Theorem 4.10. For each $i \leq k$, let $T_{i} \subset K_{\gamma}$ denote the image in $K_{\gamma}$ of a minimum weight spanning tree of $K_{\gamma-\alpha_{i}}$ under the cover morphism $\psi_{i}: K_{\gamma-\alpha_{i}} \rightarrow K_{\gamma}$. Additionally, let

$$
E^{\prime}=\mathcal{H}\left(\left\{(z, w): z_{1} \alpha_{1}+\cdots+z_{k} \alpha_{k}=w_{1} \alpha_{1}+\cdots+w_{k} \alpha_{k}\right\}\right) \cap E\left(K_{\gamma}\right)
$$

be the elements of the Graver basis of $\Gamma$ that lie in $\mathrm{Z}(\gamma) \times \mathrm{Z}(\gamma)$. The graph

$$
G^{\prime}=\left(\mathrm{Z}(\gamma), E\left(T_{1}\right) \cup \cdots \cup E\left(T_{k}\right) \cup E^{\prime}\right)
$$

is connected, and any minimum weight spanning tree of $G^{\prime}$ is minimum weight in $K_{\gamma}$.

Proof. Fix $z, w \in \mathbf{Z}(\gamma)$. If $j \in \operatorname{supp}(z) \cap \operatorname{supp}(w)$, then a path in $T_{j}$ connects $z$ and $w$. Alternatively, if $z$ and $w$ have disjoint support and $(z, w) \notin E^{\prime}$, then $(z, w)$ can be expressed as a $\operatorname{sum}(z, w)=\left(z_{1}, z_{1}^{\prime}\right)+\cdots+\left(z_{r}, z_{r}^{\prime}\right)$ of Graver basis elements, so the chain $q$ given by

$$
z_{1}+z_{2}+\cdots+z_{r}, \quad z_{1}^{\prime}+z_{2}+\cdots+z_{r}, \quad \ldots, \quad z_{1}^{\prime}+z_{2}^{\prime}+\cdots+z_{r}^{\prime}
$$

connects $z$ and $w$ in $K_{\gamma}$. Since each edge in $q$ connects factorizations with common support, $z$ and $w$ are connected in $G^{\prime}$.

Next, let $T^{\prime}$ be any minimum weight spanning tree of $G^{\prime}$. It suffices to prove that the unique path $q^{\prime}$ in $T^{\prime}$ connecting $(z, w) \in E\left(K_{\gamma}\right) \backslash E\left(T^{\prime}\right)$ has weight at most $\mathrm{d}(z, w)$. 
If $j \in \operatorname{supp}(z) \cap \operatorname{supp}(w)$, then the unique path from $z$ to $w$ in $T_{j}$ has weight at most $\mathrm{d}(z, w)$, and thus so does $q^{\prime}$. On the other hand, if $z$ and $w$ have disjoint support, then the $i$-th edge in the chain $q$ constructed in the above paragraph has weight

$$
\mathrm{d}\left(z_{i}, z_{i}^{\prime}\right)=\max \left(\left|z_{i}\right|,\left|z_{i}^{\prime}\right|\right) \leq \max (|z|,|w|)=\mathrm{d}(z, w)
$$

meaning $q^{\prime}$ has weight at $\operatorname{most} \max \left\{\mathrm{d}\left(z_{i}, z_{i}^{\prime}\right): i \leq r\right\} \leq \mathrm{d}(z, w)$. This completes the proof.

We are now ready to state Algorithm 4.11.

Algorithm 4.11. Finds $\mathrm{c}(\gamma)$ for any affine semigroup element $\gamma \in \Gamma=\left\langle\alpha_{1}, \ldots, \alpha_{k}\right\rangle \subset \mathbb{N}^{d}$.

function CatenaryDegreeOfAffineSemigroupElement $(\gamma, \Gamma)$

$T \leftarrow$ MinimumWeightSpanningTree $(\gamma, \Gamma)$

return $\max \{\mathrm{d}(z, w):(z, w) \in E(T)\}$.

end function

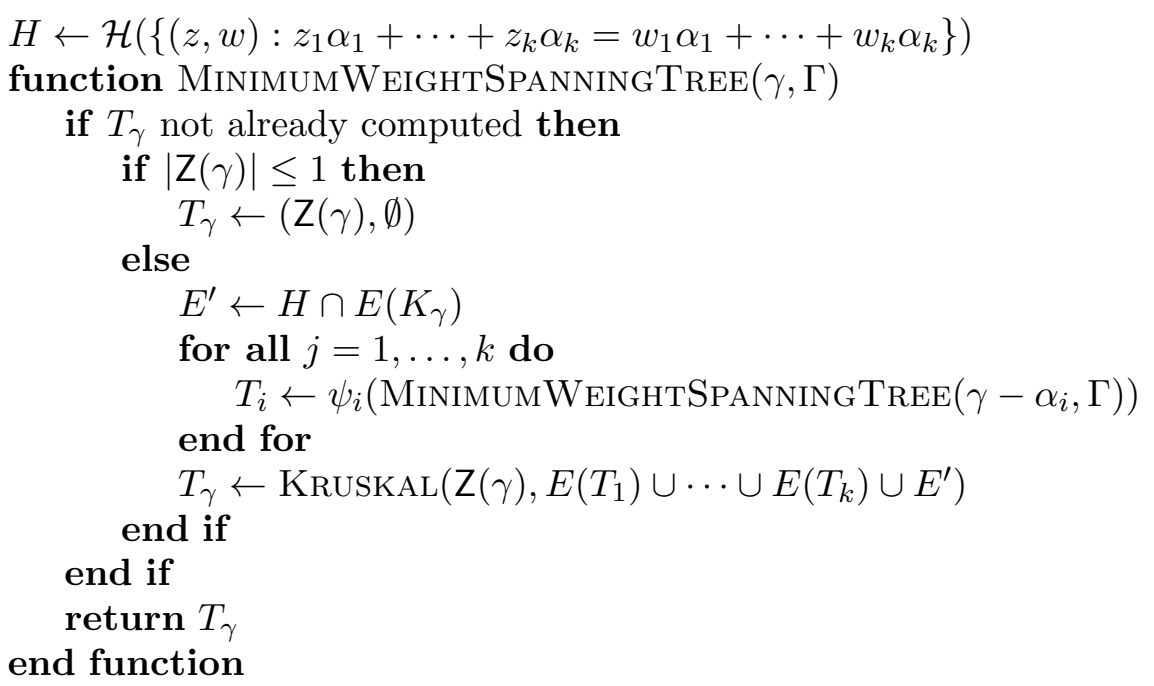

Theorem 4.12. For any affine semigroup element $\gamma \in \Gamma$, Algorithm 4.11 returns $\mathrm{c}(\gamma)$.

Proof. Proposition 4.6 and Theorem 4.10 ensure that MinimumWeightSpanningTree returns a minimum weight spanning tree $T \subset K_{\gamma}$, and by Lemma 4.5, the maximum weight among the edges of $T$ equals $\mathrm{c}(\gamma)$.

4.1. Implementation and benchmarks. Algorithm 4.11 has now been implemented as CatenaryDegreeElementListWRTNumericalSemigroup in the numericalsgps [14] GAP [17] package. The function definition mirrors that of the dynamic length sets and $\omega$-primality functions [3], which motivated the results in this section. A version for affine semigroups is forthcoming, pending discussion of the function definition. The code is accessible in [14] or from the official GAP web page http://www.gap-system.org. The development version of numericalsgps can be found in https://bitbucket.org/gap-system/numericalsgps.

We give a few implementation notes before concluding the section with an example. 
1. If $\Gamma=\left\langle\alpha_{1}, \ldots, \alpha_{k}\right\rangle \subset \mathbb{N}$ is a numerical semigroup, then it is natural to begin with $\gamma=0$ and compute $\mathrm{c}(\gamma)$ in order. This allows the spanning trees to be stored in a ring buffer of size $\alpha_{k}$ (the largest generator), since $\gamma-\alpha_{k}$ is the smallest element that needs to be recalled to compute $\mathrm{c}(\gamma)$.

2. By storing the list of edges in $E\left(T_{\gamma}\right)$ ordered by weight and computing the union $E\left(T_{1}\right) \cup \cdots \cup E\left(T_{k}\right) \cup E^{\prime}$ via a merge of sorted lists, the first (and most computationallyintensive) step in Kruskal's Algorithm runs in linear time.

3. At no point must $\mathbf{Z}(\gamma)$ be explicitly computed. The base case $Z(\gamma) \leq 1$ consists of precisely those elements for which $E\left(T_{1}\right), \ldots, E\left(T_{k}\right)$, and $E^{\prime}$ are all empty, and in all other cases, $\mathrm{Z}(\gamma)$ is simply the set of edge endpoints by Theorem 4.10. This is essentially [3, Algorithm 3.3] for dynamically computing sets of factorizations.

Example 4.13. Given here are runtimes for three separate methods of computing $\mathrm{c}(\gamma)$ in $\Gamma=\langle 11,23,27,31,43\rangle \subset \mathbb{N}$ for $\gamma \leq 500$.

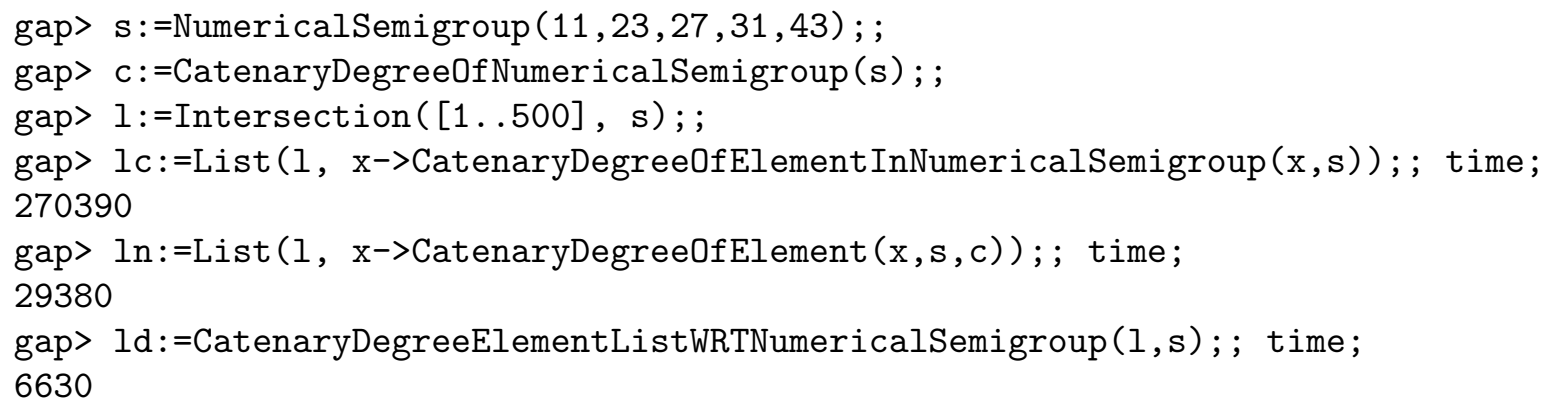

The function CatenaryDegreeOfElement InNumericalSemigroup (original implementation in the package) computes $\mathrm{c}(\gamma)$ by computing the complete graph $K_{\gamma}$ and then removing edges by weight, whereas the function CatenaryDegreeOfElement constructs a spanning tree for $K_{\gamma}$ using Kruskal's Algorithm (as discussed immediately following Definition 4.3). The latter will replace the implementation of the former in the next release of the numericalsgps package. Although CatenaryDegreeOfElement is an improvement over the original implementation, the dynamic algorithm is still considerably faster for large $\gamma$.

\section{The TAME DEgReE}

A variant of the catenary degree, the tame degree also uses distance (as in Definition 4.1) to measure how wild the factorizations of monoid elements are [22]. In this section, we present Algorithm 5.10, an improved method for computing the tame degree of a full affine semigroup (Definition 5.5). We begin by presenting Theorem 5.2, the main new theoretical result used in Algorithm 5.10, followed by a discussion of the algorithm. One of the primary motivations for having a dedicated algorithm for this family of affine semigroups is to examine the tame degree of block monoids; in Examples 5.15,5.17, we apply our algorithm in this setting.

Definition 5.1. Fix $\Gamma=\left\langle\alpha_{1}, \ldots, \alpha_{k}\right\rangle \subset \mathbb{N}^{d}$. Given $\gamma \in \Gamma$ and $i \leq k$ such that $\gamma-\alpha_{i} \in \Gamma$, the tame degree of $\gamma$ with respect to $\alpha_{i}$, denoted $\mathrm{t}_{i}(\gamma)$, is defined as the least $N \in \mathbb{N} \cup\{\infty\}$ 
such that for every $z \in \mathbf{Z}(\gamma)$, there exists $w \in \mathbf{Z}(\gamma)$ with $w_{i}>0$ and $\mathrm{d}(z, w) \leq N$. The tame degree of $\Gamma$ with respect to $\alpha_{i}$ is then defined as

$$
\mathrm{t}_{i}(\Gamma)=\sup \left\{\mathrm{t}_{i}(\gamma): \gamma-\alpha_{i} \in \Gamma\right\} .
$$

Lastly, the tame degree of $\Gamma$ is defined as $\mathrm{t}(\Gamma)=\max \left\{\mathrm{t}_{i}(\Gamma): i \leq k\right\}$.

In what follows, we denote by ' $\leq$ ' the usual partial ordering in $\mathbb{N}^{k}$, that is, $x \leq y$ if $y-x \in \mathbb{N}^{k}$, or, equivalently, if $x_{i} \leq y_{i}$ for all $i \leq k$. Notice that $x \leq y$ is also equivalent to $y \in x+\mathbb{N}^{k}=\left\{x+z: z \in \mathbb{N}^{k}\right\}$.

Theorem 5.2. Let $\Gamma=\left\langle\alpha_{1}, \ldots, \alpha_{k}\right\rangle \subset \mathbb{N}^{d}$, and fix $i \leq k$. We have

$$
\mathrm{t}_{i}(\Gamma)=\sup \left\{\mathrm{t}_{i}(\gamma): \gamma \in M_{i}\right\}
$$

where $M_{i}=\left\{\varphi_{\Gamma}(x): x \in\right.$ Minimals $\left.\leq \mathbf{Z}\left(\alpha_{i}+\Gamma\right)\right\}$.

Proof. Since $M_{i} \subseteq \Gamma$, clearly $\sup \left\{\mathrm{t}_{i}(m): m \in M_{i}\right\} \leq \mathrm{t}(\Gamma)$. Conversely, fix $\gamma \in \alpha_{i}+\Gamma$ and $z \in \mathbf{Z}(\gamma)$. If $z_{i}>0$, then choose $w=z$ in the definition of tame degree. Otherwise, $z \in \mathbf{Z}\left(\alpha_{i}+\Gamma\right)$ since $\gamma \in \alpha_{i}+\Gamma$, and consequently, there exists $z^{\prime} \in$ Minimals $\leq \mathbf{Z}\left(\alpha_{i}+\Gamma\right)$ such that $z^{\prime} \leq z$. Set $\gamma^{\prime}=\varphi_{\Gamma}\left(z^{\prime}\right)$. By the definition of $\mathrm{t}_{i}(-)$, there exists $w^{\prime} \in \mathbf{Z}\left(\gamma^{\prime}\right) \cap\left(\alpha_{i}+\mathbb{N}^{k}\right)$ with $\mathrm{d}\left(z^{\prime}, w^{\prime}\right) \leq \mathrm{t}_{i}\left(\gamma^{\prime}\right)$. Let $w=w^{\prime}+\left(z-z^{\prime}\right)$. Upon verifying that

$$
\varphi_{\Gamma}(w)=\varphi_{\Gamma}\left(w^{\prime}\right)+\varphi_{\Gamma}\left(z-z^{\prime}\right)=\varphi_{\Gamma}\left(z^{\prime}\right)+\varphi_{\Gamma}\left(z-z^{\prime}\right)=\varphi_{\Gamma}(z)=\gamma,
$$

$\mathrm{d}(z, w)=\mathrm{d}\left(z^{\prime}, w^{\prime}\right) \leq \mathrm{t}_{i}\left(\gamma^{\prime}\right)$, and $w \in \mathrm{Z}(\gamma) \cap\left(\alpha_{i}+\mathbb{N}^{k}\right)$, it follows that $\mathrm{t}_{i}(n) \leq \mathrm{t}_{i}(m)$. This implies $\mathrm{t}_{i}(\gamma) \leq \sup \left\{\mathrm{t}_{i}\left(\gamma^{\prime}\right): \gamma^{\prime} \in M_{i}\right\}$, and consequently $\mathrm{t}_{i}(\Gamma) \leq \sup \left\{\mathrm{t}_{i}\left(\gamma^{\prime}\right): \gamma^{\prime} \in M_{i}\right\}$.

Remark 5.3. Theorem 5.2 holds in more generality with a nearly identical proof; the only requirement is that $\Gamma$ be atomic. We specialize to the affine setting here to simplify notation since Algorithm 5.10 requires this assumption; see [22] for the general definitions.

Remark 5.4. Comparing Theorem 5.2 to [4, Proposition 3.3], it is not a coincidence that the elements needed to compute the tame degree of $\Gamma$ are the same as those needed to compute the $\omega$-primality invariant (see [4, Definition 3.1]). Some evidence of this connection was already observed for numerical semigroups; see, for instance, [7, Corollary 3] and [4, Remark 5.9].

Arranging the atoms $\alpha_{1}, \ldots, \alpha_{k}$ of $\Gamma$ as the columns of a matrix $A$, the set $\mathrm{Z}(\gamma)$ of factorizations of $\gamma \in \Gamma$ coincides with the nonnegative integer solutions to the linear system

$$
A x=\gamma \text {. }
$$

Prior to Theorem 5.2, the tame degree of $\Gamma$ was typically computed by first computing the Graver basis of $A$ [9]. We propose here an alternative method, instead computing the sets

$$
\mathcal{M}_{i}=\text { Minimals }_{\leq} \mathrm{Z}\left(\alpha_{i}+\Gamma\right) \quad \text { and } \quad M_{i}=\left\{\varphi_{\Gamma}(z): z \in \mathcal{M}_{i}\right\}
$$

for each $i \in\{1, \ldots, k\}$. By Dickson's lemma, both $\mathcal{M}_{i}$ and $M_{i}$ have finitely many elements.

For the calculation of $\mathcal{M}_{i}$ one can use [32, Algorithm 16] (this is precisely how $\omega$-primality is computed in [19]). However, it turns out that Normaliz [6, 5] performs this task faster. To obtain $\mathcal{M}_{i}$, we first compute the minimal nonnegative integer solutions of

$$
(A \mid-A)\left(\begin{array}{l}
x \\
y
\end{array}\right)=\alpha_{i},
$$


and then project on the first $k$ coordinates (here, $(A \mid-A)$ is the matrix having as columns the columns of $A$ followed by those of $-A$ ). From the resulting (finite) set, we simply take those elements that are minimal with respect to $\leq$.

Since the Graver basis of $A$ coincides with the minimal nonnegative integer solutions of

$$
(A \mid-A)\left(\begin{array}{l}
x \\
y
\end{array}\right)=0
$$

it would seem that our approach has no significant computational advantage over the procedure given in [9]. However, combining with [4, Corollary 3.5] (stated here as Proposition 5.7) yields significant performance improvements for full affine semigroups.

Definition 5.5. An affine semigroup $\Gamma \subset \mathbb{N}^{d}$ is full if $\mathrm{G}(\Gamma) \cap \mathbb{N}^{d}=\Gamma$, where $\mathrm{G}(\Gamma)$ denotes the subgroup of $\mathbb{Z}^{d}$ generated by $\Gamma$.

Remark 5.6. Up to isomorphism, every full affine semigroup is a (reduced finitely generated) Krull monoid [25, Proposition 2]. Also, the class of reduced Krull monoids coincides with the class of saturated submonoids of free monoids [22, Theorem 2.4.8].

Proposition 5.7 ([4, Corollary 3.5]). If $\Gamma=\left\langle\alpha_{1}, \ldots, \alpha_{k}\right\rangle \subset \mathbb{N}^{d}$ is full, then for every $\gamma \in \Gamma$,

$$
\mathrm{Z}(\gamma+\Gamma)=\left\{x \in \mathbb{N}^{k}: \gamma \leq \varphi_{\Gamma}(x)\right\}
$$

Example 5.8. The "full" hypothesis in Proposition 5.7 cannot be omitted. For example, the numerical semigroup $\Gamma=\langle 3,5,7\rangle \subset \mathbb{N}$ is not full since $5-3=2 \in(\mathrm{G}(\Gamma) \cap \mathbb{N}) \backslash \Gamma$ (in fact, the only full numerical semigroup is $\mathbb{N}$ ). In this example, we see that

$$
\text { Minimals } \leq \mathbf{Z}(3+\Gamma)=\{(0,0,2),(0,1,1),(0,2,0),(1,0,0)\},
$$

even though Minimals $\leq\left\{x \in \mathbb{N}^{3}: 3 x_{1}+5 x_{2}+7 x_{3} \geq 3\right\}=\{(1,0,0),(0,1,0),(0,0,1)\}$.

Recalling $A=\left(\alpha_{1}|\cdots| \alpha_{k}\right)$ from above, Proposition 5.7 states that

$$
\mathrm{Z}(\gamma+\Gamma)=\left\{x \in \mathbb{N}^{k}: A x \geq \gamma\right\},
$$

meaning that $\mathcal{M}_{i}$ can be computed as a Hilbert basis. At this point, in order to compute $\mathrm{t}_{i}(\Gamma)$ using Theorem 5.2, it remains to compute $\mathrm{t}_{i}(\gamma)$ for each $\gamma \in M_{i}$. To this end, Proposition 5.9 implies that for fixed $z \in$ Minimals $\leq \mathrm{Z}\left(\alpha_{i}+\Gamma\right)$ with $z_{i}=0$, we can find the closest factorization $w \in \mathbb{N}^{k}$ to $z$ in the fiber of $\varphi_{\Gamma}(z)$ with $w_{i} \neq 0$ by minimizing $|w|$ subject to the constraints $A w=A z$ and $w \cdot z=0$. In particular, this expresses $\mathrm{t}_{i}(\gamma)$ as the result of an integer linear programming problem.

Proposition 5.9. Let $\Gamma=\left\langle\alpha_{1}, \ldots, \alpha_{k}\right\rangle \subset \mathbb{N}^{d}$, fix $i \leq k$, and fix $z \in \operatorname{Minimals}_{\leq} \mathrm{Z}\left(\alpha_{i}+\Gamma\right)$. If $z_{i}=0$ and $w \in \mathbb{N}^{k}$ fulfills $w_{i} \neq 0$ and $A w=A z$, then $z \cdot w=0$ and $\mathrm{d}(z, w)=\max \{|z|,|w|\}$.

Proof. Assume to the contrary that $u=\operatorname{gcd}(z, w) \neq 0$. As $z_{i}=0$, we deduce that $u_{i}=0$. This implies that the $i$-th coordinate of $w-u$ is nonzero, and consequently $w-u \in \mathbf{Z}\left(\alpha_{i}+\Gamma\right)$. Hence $z-u \in \mathrm{Z}\left(\alpha_{i}+\Gamma\right)$ and $z-u<z$, contradicting the minimality of $z$.

The last necessary observation is that $e_{i} \in$ Minimals $\leq \mathrm{Z}\left(\alpha_{i}+\Gamma\right)$, where $e_{i}$ is the $i$ th column

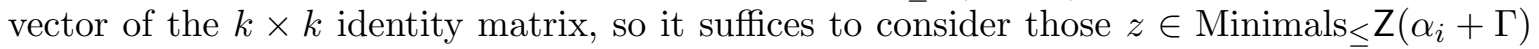
satisfying $z_{i}=0$ when computing $\mathrm{t}_{i}(\Gamma)$. We now summarize our procedure in Algorithm 5.10 . 


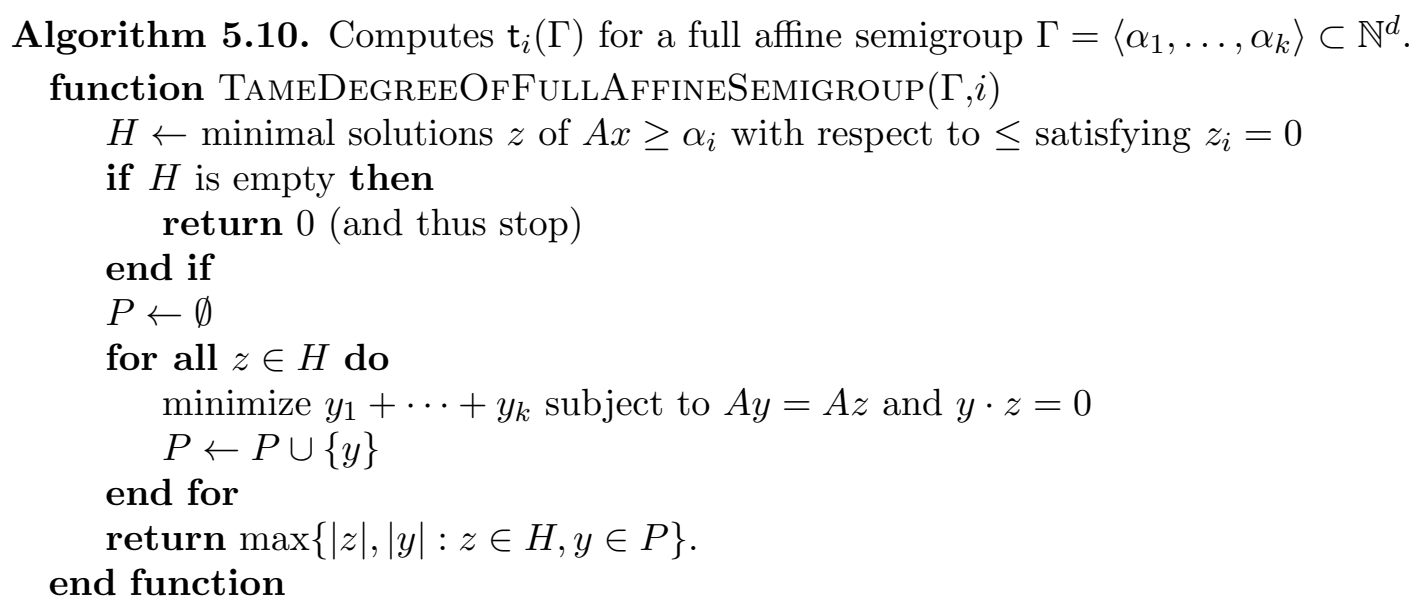

Theorem 5.11. For any full affine semigroup $\Gamma$, Algorithm 5.10 returns $\mathrm{t}_{i}(\Gamma)$.

Proof. Theorem 5.2, Propositions 5.7 and 5.9, and the discussion in between.

Remark 5.12. Notice that if all solutions $y$ obtained in Algorithm 5.10 satisfy $|y| \leq|z|$, then $\mathrm{t}_{i}(\Gamma)$ will coincide with the $\omega$-primality of $\alpha_{i}, \omega\left(\alpha_{i}\right)$ (see [4, Proposition 3.3]). Indeed, this provides an alternative proof that $\omega\left(\alpha_{i}\right) \leq \mathrm{t}_{i}(\Gamma)$.

5.1. Implementation and benchmarks. We have implemented Algorithm 5.10 in the numericalsgps [14] GAP [17] package. The function TameDegreeOfAffineSemigroup therein has two main methods: one for affine semigroups in general, and another for full affine semigroups (affine semigroups with the attribute HasEquations). The code is accessible in [14] or from the official GAP web page http://www.gap-system.org. The development version of numericalsgps can be found in https://bitbucket.org/gap-system/numericalsgps.

We conclude this section by applying our implementation of Algorithm 5.10 in several examples of block monoids (Definition 5.13).

Definition 5.13. Fix an abelian group $(G,+)$ and a finite subset $H=\left\{g_{1}, \ldots, g_{n}\right\} \subset G \backslash\{0\}$. The block monoid associated to $H$ is the affine semigroup

$$
\mathcal{B}(H)=\left\{\left(x_{1}, \ldots, x_{n}\right) \in \mathbb{N}^{n}: x_{1} g_{1}+\cdots+x_{n} g_{n}=0\right\} \subset \mathbb{N}^{n} .
$$

Denote by $\mathcal{B}(G)$ the block monoid of the set of nonzero elements of $G$.

Remark 5.14. It is easy to show that $\mathcal{B}(H)$ is a full affine semigroup. Many factorization properties of a monoid can be derived from the factorization properties of the block monoid associated to its class group (see [22] for a thorough treatment).

Example 5.15. We begin by computing the tame degree of $\Gamma=\mathcal{B}\left(\mathbb{Z}_{2}^{3}\right)$. If $B$ denotes the matrix with columns the nonzero elements of $\mathbb{Z}_{2}^{3}$, then $\Gamma$ is the submonoid of $\mathbb{N}^{7}$ of nonnegative integer solutions of the system

$$
B x \equiv 0 \bmod 2 .
$$

The tame degree of $\Gamma$ can be computed in GAP as follows. 


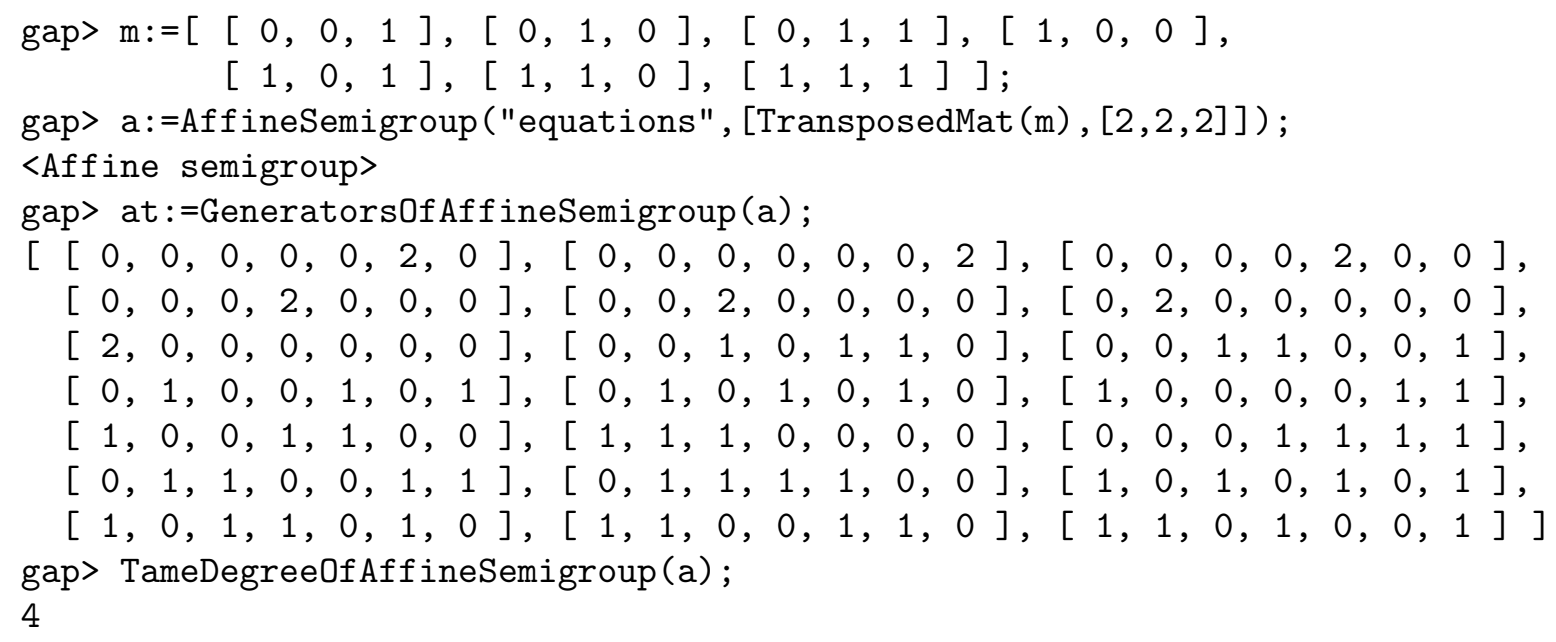

We use the package NormalizInterface 23 that utilizes the Normaliz library to compute the Hilbert bases. Although 4ti2 [1] can also be used to compute Graver and Hilbert bases via the 4ti2gap 2 package, in this example 4ti2 was slower than Normaliz. The computation of $\mathrm{t}(\Gamma)$ took $4955 \mathrm{~ms}$ on an i7 laptop with $16 \mathrm{~GB}$ of memory (Normaliz was compiled without OpenMP, and thus was running in a single thread). For comparison, an implementation of the procedure described in [9] took 1,048,757 ms.

The runtime can actually be improved further. According to [16], in order to compute $\mathrm{t}(\Gamma)$ it suffices to compute $\mathrm{t}_{i}(\Gamma)$ with $\left|\alpha_{i}\right|=4$. This is accomplished as follows, where minimalElementsPrincipalIdealOfFullAffineSemigroup implements Proposition 5.7.

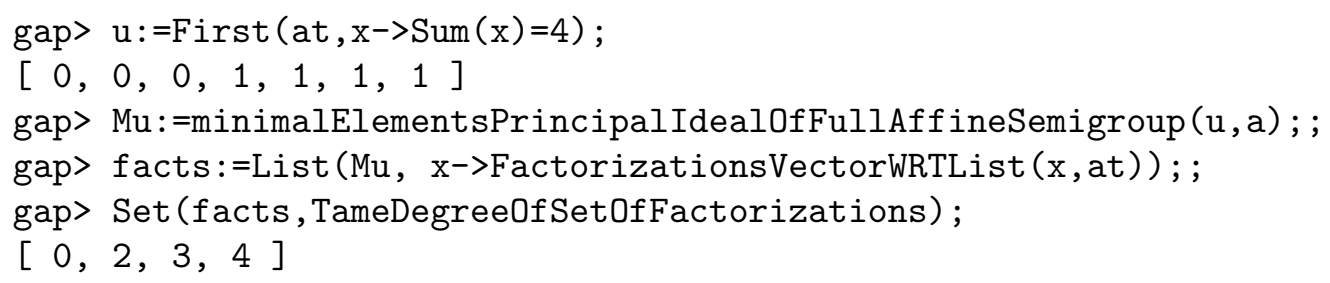

It took $2 \mathrm{~ms}$ to compute at (the set of atoms of $\Gamma$ ), and $12 \mathrm{~ms}$ to compute Mu. It took another $420 \mathrm{~ms}$ to complete the third line above, and the final line finished in $425 \mathrm{~ms}$. In total, it takes less than a second to compute the tame degree of $\Gamma$.

Example 5.16. Running Algorithm 5.10 for $\Gamma=\mathcal{B}\left(\mathbb{Z}_{2} \times \mathbb{Z}_{3}\right)$ yields $\mathrm{t}(\Gamma)=8$ after $19214 \mathrm{~ms}$; execution of the procedure presented in [9] was killed after several hours.

Example 5.17. As the size of $G$ increases, the number of atoms (i.e. the ambient dimension of $\mathcal{B}(G)$ ) quickly makes use of Algorithm 5.10 infeasible. Indeed, the block monoid $\mathcal{B}\left(\mathbb{Z}_{2}^{4}\right)$ has 323 atoms, and $\mathcal{B}\left(\mathbb{Z}_{2}^{5}\right)$ has 20367 atoms. Although Algorithm 5.10 is considerably faster than the general-purpose algorithm introduced in [9], it is still not sufficient to compute $\mathrm{t}\left(\mathcal{B}\left(\mathbb{Z}_{2}^{4}\right)\right)$.

Given below is the analysis for $\mathrm{t}\left(\mathcal{B}\left(\mathbb{Z}_{2}^{4}\right)\right)$ by using the supercomputer alhambra.ugr.es

(1) First attempt: 16 cores with 30 GB of internal memory. 

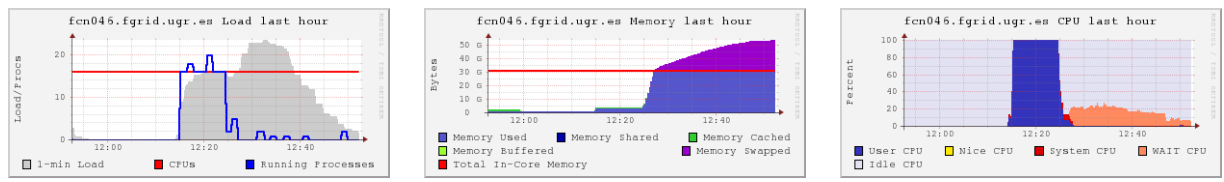

Internal memory is quickly exhausted and the system begins using swap memory, decaying the number of processors and the user CPU usage. The process is killed before it finishes.

(2) Second attempt: 32 cores with 256 GB of internal memory.
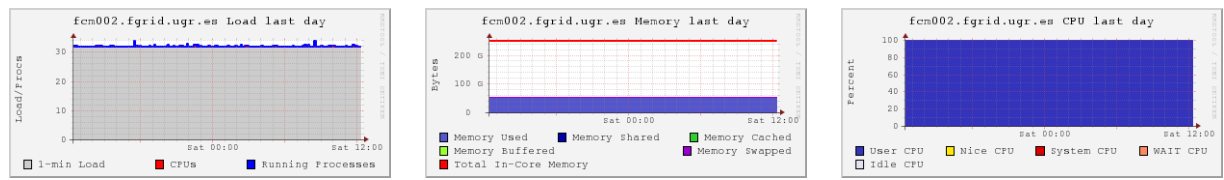

After 72 hours of execution, Mu is still not computed. We contacted W. Bruns, B. Ichim and C. Söger (developers of Normaliz) and were told (i) the input has too many variables and few equations to finish in a reasonable time, and (ii) the cone has tens of millions of extremal rays and thus requires extensive memory (> $50 \mathrm{~GB}$ ) just to store.

The point of this example is that even though we may consider $\mathbb{Z}_{2}^{4}$ a "small" group, the monoid $\mathcal{B}\left(\mathbb{Z}_{2}^{4}\right)$ has 20367 atoms, and this translates to working in an ambient space of dimension 323! Thus, Algorithm 5.10 will fail to compute the tame degree for this monoid with the computational tools at hand.

\section{AcKnowledgements}

Supported by FQM-343, FQM-5849 and FEDER funds. The authors would like to thank Winfried Bruns, Alfred Geroldinger, Alfredo Sánchez-R.-Navarro, Richard Sieg and Christof Söger for their comments and discussions on Section5. Additionally, we thank Winfried Bruns for pointing out that we only need a single Hilbert basis computation in Algorithm 3.6, as well as for his assistance with several computations in Normaliz. The first author also thanks the Centro de Servicios de Informática y Redes de Comunicaciones (CSIRC), Universidad de Granada, for providing the computing time, especially Rafael Arco Arredondo for his personal time advising which queues should be used in the experiments, and for installing everything needed on the supercomputer alhambra.ugr.es.

\section{REFERENCES}

[1] The 4ti2 team, 4ti2-a software package for algebraic, geometric and combinatorial problems on linear spaces, http://www.4ti2.de.

[2] P.A. García-Sánchez and A. Sánchez-R.-Navarro, 4ti2gap, GAP wrapper for 4ti2, https:// bitbucket.org/gap-system/4ti2gap

[3] T. Barron, C. O'Neill, and R. Pelayo, On dynamic algorithms for factorization invariants in numerical monoids, to appear, Mathematics of Computation. Available at arXiv: math.AC/1507.07435.

[4] V. Blanco, P.A. García-Sánchez and A. Geroldinger, Semigroup theoretical characterizations of arithmetical invariants with applications to numerical monoids and Krull monoids, Illinois Journal of Mathematics, 55 (2011), 1385-1414.

[5] W. Bruns, B. Ichim, T. Römer and C. Söger, Normaliz, Algorithms for rational cones and affine monoids, http://www.math.uos.de/normaliz. 
[6] W. Bruns, B. Ichim and C. Söger, The power of pyramid decompositions in Normaliz, to appear, J. Symb. Comput. Available at arXiv:1206.1916.

[7] S. T. Chapman, P.A. García-Sánchez, and D. Llena, The catenary and tame degree of numerical monoids, Forum Mathematicum 21(2009), 117-129.

[8] S. T. Chapman, P. A. Garca-Snchez, D. Llena, A. Malyshev, D. Steinberg, On the Delta set and the Betti elements of a BF-monoid, Arab J. Math 1 (2012), 53-61.

[9] S.T. Chapman, P.A. García-Sánchez, D. Llena, V. Ponomarenko, and J.C. Rosales, The catenary and tame degree in finitely generated commutative cancellative monoids, Manuscr. Math. 120 (2006), 253-264.

[10] S. Chapman, M. Corrales, A. Miller, C. Miller, and D. Patel, The catenary and tame degrees on a numerical monoid are eventually periodic, J. Aust. Math. Soc. 97 (2014), no. 3, 289-300.

[11] Costantini, M. and de Graaf, W., singular, The GAP interface to Singular, Version 12.04.28 (2012), (GAP package), http://www.gap-system.org/HostedGapPackages/singular/.

[12] D. Cox, J. Little, and D. O'Shea, Ideals, varieties, and algorithms: An introduction to computational algebraic geometry and commutative algebra, 4th edition, Undergraduate Texts in Mathematics, Springer, Cham, 2015.

[13] W. Decker, G.-M. Greuel, G. Pfister, H. Schönemann, Singular 4-1-0 - A computer algebra system for polynomial computations. http://www.singular.uni-kl.de (2016).

[14] Delgado, M., P. A. García-Sánchez and J. Morais, NumericalSgps, a package for numerical semigroups, Version 1.0.1 (2015), (Refereed GAP package), http://www.gap-system.org.

[15] G. Gamrath, T. Fischer, T. Gally, A. M. Gleixner, G.Hendel, T. Koch, S. J. Maher, M. Miltenberger, B. Müller, M. E. Pfetsch, C. Puchert, D. Rehfeldt, S. Schenker, R. Schwarz, F. Serrano, Y. Shinano, S. Vigerske, D. Weninger, M. Winkler, J. T. Witt, J. Witzig, The SCIP Optimization Suite 3.2, ZIB-Report (15-60) (2016).

[16] W. Gao, A. Geroldinger and W.A. Schmid, Local and global tameness in Krull monoids, Communications in Algebra, 43:262-296, 2015.

[17] The GAP Group, GAP - Groups, Algorithms, and Programming, 2014, http://www. gap-system.org

[18] P. García-Sánchez, D. Llena, and A. Moscariello, Delta sets for numerical semigroups with embedding dimension three, preprint. Available at arXiv: math.AC/1504.02116.

[19] J. I. García-García, M. A. Moreno-Frías, and A. Vigneron-Tenorio, Computation of the $\omega-$ primality and asymptotic $\omega$-primality, applications to numerical semigroups, Israel J. Math. 206 (2015), no. 1, 395-411. Available from arXiv:1307.5807.

[20] P. García-Sánchez, An overview of the computational aspects of nonunique factorization invariants, preprint. Available at arXiv: math.AC/1504.07424.

[21] P. A. García-Sánchez, I. Ojeda, A. Sánchez-R.-Navarro, Factorization invariants in half-factorial affine semigroups, Internat. J. Algebra Comput. 23 (2013), 111-122.

[22] A. Geroldinger and F. Halter-Koch, Non-Unique Factorizations: Algebraic, Combinatorial, and Analytic Theory, Chapman and Hall/CRC, Boca Raton, Florida, 2006.

[23] Gutsche, S., Horn, M. and Söger, C., NormalizInterface, GAP wrapper for Normaliz, Version 0.9.5 (2016), (GAP package), https://gap-packages.github.io/NormalizInterface

[24] J. Herzog, Generators and relations of abelian semigroups and semigroup rings, Manuscripta Math. 3 (1970), 175-193.

[25] F. Kainrath, G. Lettl, Geometric notes on monoids, Semigroup Forum 61 (2000), 298-302.

[26] J. B. Kruskal, On the shortest spanning subtree of a graph and the traveling salesman problem, Proceedings of the American Mathematical Society 7 (1956), 48-50.

[27] C. O'Neill, On factorization invariants and Hilbert functions, preprint. Available at arXiv: math.AC/1503.08351.

[28] C. O'Neill and R. Pelayo, Factorization invariants in numerical monoids, to appear, Contemp. Math. Available at arXiv: math.AC/1508.00128.

[29] L. Rédei, The theory of finitely generated commutative semigroups, Pergamon, OxfordEdinburgh-New York, 1965. 
[30] J.C. Rosales and P.A. García-Sánchez, Finitely Generated Commutative Monoids, Nova Science Publishers, 1999.

[31] J. Rosales and P. García-Sánchez, Numerical semigroups, Developments in Mathematics, Vol. 20, Springer-Verlag, New York, 2009.

[32] J. C. Rosales, P. A. García-Sánchez, and J. I. García-García, Irreducible ideals of finitely generated commutative monoids, J. Algebra 238 (2001), 328-344.

[33] The Sage Developers, SageMath, the Sage Mathematics Software System (Version 7.2), 2016, http://www.sagemath.org.

Departamento de Álgebra, Universidad de Granada, 18071 Granada, España

E-mail address: pedro@ugr.es

Mathematics, University of California, Davis, One Shields Ave, Davis, CA 95616

E-mail address: coneill@math.ucdavis.edu

Mathematics Department, University of Oregon, Eugene, OR 97403

E-mail address: gwebb@uoregon.edu 